\title{
Vitamin B Complex
}

National Cancer Institute

\section{Source}

National Cancer Institute. Vitamin B Complex. NCI Thesaurus. Code C940.

A compound containing several or all of the B-vitamins, including thiamine, riboflavin, niacin (nicotinic acid), niacinamide (nicotinamide), the vitamin B6 group (including pyridoxine, pyridoxal, pyridoxamine), biotin, pantothenic acid, folic acid, possibly paraaminobenzoic. 